\title{
New hydrogen-deficient (pre-) white dwarfs in the Sloan Digital Sky Survey Data Release 10
}

\author{
K. Werner ${ }^{1}$, T. Rauch ${ }^{1}$, and S. O. Kepler ${ }^{2}$ \\ ${ }^{1}$ Institute for Astronomy and Astrophysics, Kepler Center for Astro and Particle Physics, Eberhard Karls University, Sand 1, \\ 72076 Tübingen, Germany \\ e-mail: werner@astro.uni-tuebingen.de \\ 2 Instituto de Física, Universidade Federal do Rio Grande do Sul, 91501-900 Porto-Alegre, RS, Brazil
}

Received 16 January 2014 / Accepted 12 March 2014

\begin{abstract}
We have detected 21 new, very hot hydrogen-deficient (pre-) white dwarfs (spectral types O(He), hot DO, PG1159) in the Data Release 10 of the Sloan Digital Sky Survey. We present the results of non-local thermodynamic equilibrium (non-LTE) model-atmosphere analyses. The seven PG1159 stars have effective temperatures and surface gravities in the ranges $T_{\text {eff }}=100000-140000 \mathrm{~K}$ and $\log g=6-7.5$. The hottest one is a new member of the rare hybrid-PG1159 subtype, indicating the presence of residual hydrogen. The ten new hot DOs span $T_{\text {eff }}=65000-120000 \mathrm{~K}$ and $\log g=7.5-8$. Carbon abundances were derived and it is found that the PG1159 star values $(\mathrm{C} / \mathrm{He}=0.33-0.03$ by number $)$ and the $\mathrm{DO}$ values $(<0.0004-0.01)$ cover distinct but adjacent ranges. With the discovery of four new $\mathrm{O}(\mathrm{He})$ stars $\left(T_{\text {eff }}=80000-100000 \mathrm{~K}, \log g=5-5.5, \mathrm{C} / \mathrm{He}=0.002-0.01\right)$, we double the number of known members of this rare spectral class. One of them is particularly interesting because it is unusually rich in $\mathrm{C}, \mathrm{N}, \mathrm{O}$, and $\mathrm{Si}$. Unexpected problems arose with the DOs because many He II lines of every other star are so deep that they cannot be matched by any model. This is most conspicuous in the case of one object which exhibits the occurrence of ultrahigh-ionization metal features, a hitherto unexplained phenomenon that is known from a disquieting large fraction of hot DOs.
\end{abstract}

Key words. stars: abundances - stars: atmospheres - stars: evolution - stars: AGB and post-AGB - white dwarfs stars: fundamental parameters

\section{Introduction}

PG 1159 stars are hot hydrogen-deficient post-Asymptotic Giant Branch (post-AGB) stars (Werner \& Herwig 2006). In the Hertzsprung-Russell diagram, they cover a region comprising the hottest central stars of planetary nebulae and white dwarfs ( $\left.T_{\text {eff }}=75000-200000 \mathrm{~K}, \log g=5.5-8\right)$. Their H-deficiency is most probably the result of a (very) late He-shell flash. Their envelopes are mainly composed of $\mathrm{He}, \mathrm{C}$, and $\mathrm{O}$, with rather diverse abundance patterns $(\mathrm{He}=0.30-0.85, \mathrm{C}=0.13-0.60, \mathrm{O}=$ $0.02-0.20$, mass fractions). The spectra are dominated by lines from He II and C IV.

DO white dwarfs (WDs) are hot, He-rich white dwarfs. They exhibit lines from He I and He II (corresponding to $T_{\text {eff }} \gtrsim$ $50000 \mathrm{~K})$, or, He II only $\left(T_{\text {eff }} \gtrsim 70000 \mathrm{~K}\right)$. The latter are called hot DOs and are found with temperatures up to about $120000 \mathrm{~K}$ (e.g. Dreizler \& Werner 1996; Hügelmeyer et al. 2006) ${ }^{1}$. Here we confine our search and analysis to such hot DOs. Temperatures and gravities of PG 1159 stars and hot DOs overlap, and some DOs have trace amounts of carbon (of the order $\mathrm{C} / \mathrm{He}=0.01$ by number). It is therefore thought that both classes form an evolutionary sequence. A cooling PG 1159 star turns into a DO white dwarf when gravitational settling removes heavy elements from the photosphere, unless it is of subtype hybridPG 1159 having residual hydrogen in the atmosphere. In that case a hydrogen-rich (DA) white dwarf will emerge. In view of

\footnotetext{
1 We exclude KPD 0005+5106 which was classified as DO but actually is a pre-WD $\left(T_{\mathrm{eff}}=200000 \mathrm{~K}, \log g=6.7\right.$; Wassermann et al. 2010).
}

the large number of known WDs, the number of PG 1159 stars and hot DOs is small. It is rewarding to study each of these stars individually, because occasionally they have surprising characteristics. The most recent example is the first-time detection of krypton and xenon as well as many other trans-iron elements in the hot DO RE 0503-289 (Werner et al. 2012). The large overabundance of these elements allows us to study in detail the PG 1159-DO transformation process. In addition, the enriched atmosphere of this (and other hot DOs and PG 1159 stars) is used as a plasma laboratory in which atomic data of highly ionized species can be investigated (e.g. Rauch et al. 2012, 2013). In the present study we also find in several objects spectral lines that have not been detected before in $\mathrm{H}$-deficient stars.

In the Data Release (DR) 4 of the Sloan Digital Sky Survey (SDSS), Hügelmeyer et al. (2005, 2006) discovered many new PG 1159 stars and DOs and performed spectral analyses. Here we present a similar study for the DR 10. Kleinman et al. (2013) already announced the identification of a number of new DOs and PG 1159s in DR 7. In addition, we have now searched DR 10 for such hitherto unidentified stars. From the 21 stars presented in this paper, ten were already included in the DR 7.

During our analysis of the selected stars, four of them turned out to be $\mathrm{O}(\mathrm{He})$ stars. Stars of this class are defined as being hot helium-dominated pre-WDs exhibiting He II lines. In contrast to the DOs they have low gravity and in contrast to PG 1159 stars they have at most very weak C IV lines. The four previously know $\mathrm{O}(\mathrm{He}) \mathrm{s}$ were recently re-analyzed $\left(T_{\mathrm{eff}}=\right.$ $110000 \mathrm{~K}-135000 \mathrm{~K}, \log g=5.8-6.4, \mathrm{C} / \mathrm{He}<2 \times 10^{-4}-0.003$ by number; Reindl et al. 2014). Their origin is rather unclear, 
Table 1. Parameters of the new PG 1159 stars, $\mathrm{O}(\mathrm{He})$ stars, and hot DO white dwarfs.

\begin{tabular}{|c|c|c|c|c|c|c|}
\hline $\begin{array}{l}\text { Name } \\
\text { SDSS J }\end{array}$ & $\begin{array}{l}\text { Spectral } \\
\text { type }\end{array}$ & $\begin{array}{r}T_{\text {eff }} \\
{[\mathrm{kK}]}\end{array}$ & $\begin{array}{c}\log g \\
{\left[\mathrm{~cm} \mathrm{~s}^{-2}\right]}\end{array}$ & $\mathrm{C} / \mathrm{He}$ & $\begin{array}{c}M \\
{\left[M_{\odot}\right]}\end{array}$ & Remarks \\
\hline $152116.00+251437.46$ & PG 1159 & $140 \pm 15$ & $6.0 \pm 0.3$ & 0.17 & $0.58_{-0.04}^{+0.08}$ & hybrid PG1159; $\mathrm{H} / \mathrm{He}=1, \mathrm{O} / \mathrm{He}=0.0043$ \\
\hline $075415.12+085232.18$ & PG 1159 & $120 \pm 10$ & $7.0 \pm 0.3$ & 0.33 & $0.52_{-0.01}^{+0.02}$ & \\
\hline $141556.26+061822.43$ & PG 1159 & $120 \pm 10$ & $7.5 \pm 0.3$ & 0.11 & $0.58_{-0.05}^{+0.08}$ & \\
\hline $055905.02+633448.45$ & PG 1159 & $110 \pm 15$ & $7.5 \pm 0.5$ & 0.11 & $0.56_{-0.05}^{+0.17}$ & \\
\hline $105300.24+174932.95$ & PG 1159 & $100 \pm 10$ & $7.0 \pm 0.5$ & 0.11 & $0.50_{-0.02}^{+0.05}$ & \\
\hline $191845.01+624343.77$ & PG 1159 & $100 \pm 10$ & $7.2 \pm 0.3$ & 0.11 & $0.51_{-0.03}^{+0.04}$ & \\
\hline $123930.62+244321.75$ & PG 1159 & $100 \pm 15$ & $7.5 \pm 0.5$ & 0.03 & $0.55_{-0.04}^{+0.16}$ & \\
\hline $172854.34+361958.62$ & $\mathrm{O}(\mathrm{He})$ & $100 \pm 10$ & $5.0 \pm 0.2$ & 0.01 & $0.73_{-0.12}^{+0.14}$ & $\mathrm{~N} / \mathrm{He}=0.0018, \mathrm{O} / \mathrm{He}=0.002, \mathrm{Si} / \mathrm{He}=0.0015$ \\
\hline $171916.97+365326.70$ & $\mathrm{O}(\mathrm{He})$ & $100 \pm 20$ & $5.5 \pm 0.5$ & 0.01 & $0.53_{-0.02}^{+0.21}$ & \\
\hline $141812.50-024426.92$ & $\mathrm{O}(\mathrm{He})$ & $90 \pm 20$ & $5.5 \pm 0.5$ & 0.002 & $0.52_{-0.02}^{+0.09}$ & \\
\hline $075732.18+184329.28$ & $\mathrm{O}(\mathrm{He})$ & $80 \pm 20$ & $5.0 \pm 0.3$ & 0.002 & $0.53_{-0.03}^{+0.02}$ & \\
\hline $151215.72+065156.34$ & DO & $120 \pm 15$ & $7.5 \pm 0.3$ & 0.01 & $0.58_{-0.05}^{+0.08}$ & unusually strong He II lines \\
\hline $083610.09+160558.76$ & DO & $100 \pm 20$ & $7.5 \pm 0.5$ & $<0.0004$ & $0.55_{-0.04}^{+0.16}$ & \\
\hline $212721.52+053434.54$ & DO & $100 \pm 15$ & $7.5 \pm 0.5$ & $<0.0004$ & $0.55_{-0.04}^{+0.16}$ & \\
\hline $095630.14+224311.52$ & DO & $100 \pm 10$ & $7.8 \pm 0.5$ & 0.002 & $0.64_{-0.12}^{+0.22}$ & \\
\hline $082724.44+585851.68$ & DO & $90 \pm 20$ & $7.5 \pm 0.5$ & $<0.0004$ & $0.54_{-0.04}^{+0.16}$ & unusually strong He II lines \\
\hline $105956.01+404332.38$ & DO & $90 \pm 20$ & $7.5 \pm 0.5$ & 0.01 & $0.54_{-0.04}^{+0.16}$ & ultrahigh-ionization lines \\
\hline $094722.49+101523.62$ & DO & $85 \pm 15$ & $7.5 \pm 0.5$ & 0.002 & $0.53_{-0.03}^{+0.17}$ & unusually strong He II lines \\
\hline $081625.47-002815.48$ & DO & $80 \pm 10$ & $7.8 \pm 0.5$ & 0.01 & $0.61_{-0.10}^{+0.22}$ & \\
\hline $064400.80+372022.70$ & DO & $80 \pm 15$ & $8.0 \pm 0.3$ & 0.002 & $0.69_{-0.08}^{+0.15}$ & \\
\hline $082134.95+173919.40$ & DO & $65 \pm 10$ & $7.5 \pm 0.5$ & 0.002 & $0.51_{-0.10}^{+0.15}$ & unusually strong He II lines \\
\hline
\end{tabular}

Notes. Element abundances by number. Masses derived from evolutionary tracks in Fig. 5 .

because late He-shell flash evolutionary models do not predict that surface chemistry.

In the following, we describe the SDSS spectra of the new hot DOs, PG 1159 and $\mathrm{O}(\mathrm{He})$ stars (Sect. 2) and present our spectral analysis (Sect. 3). We conclude with a discussion of the obtained results (Sect. 4). In the text we will use abbreviated versions of the object names. Full names are given Table 1.

\section{Observations and line identifications}

We have visually scanned a color selected sample of white dwarf candidates in SDSS DR10 and looked for PG 1159 stars and hot DO white dwarfs. Both classes are defined to exhibit He II lines but not He I. PG 1159 stars are recognized by the occurrence of significantly strong C IV lines. Their hallmark is a broad absorption trough with similarly strong C IV $\lambda \lambda 4648 / 60$ and He II $\lambda 4686$. Some hot DOs also show C IV $\lambda \lambda 4648 / 60$ albeit much weaker. We found a total of 21 stars, comprising seven PG 1159 stars and ten DOs. The remaining four stars are of type $\mathrm{O}(\mathrm{He})$. Table 1 lists our program stars and we show their spectra in Figs. 1 and 2.

The seven new PG 1159 stars add to the forty objects found so far (Werner \& Herwig 2006). The new four O(He) stars double the number of such stars known (Reindl et al. 2014). As to the DO white dwarfs, only nine were hitherto known having $T_{\text {eff }} \geq 90000 \mathrm{~K}$ (Dreizler \& Werner 1996; Hügelmeyer et al. 2006). As we will demonstrate below, six of our ten DOs have such high temperatures.

All stars show the usual lines from He II and, except of three DOs, C IV. Two stars (the PG 1159 star SDSS 1521 and the $\mathrm{O}(\mathrm{He})$ star SDSS 1728) show additional lines from nitrogen, oxygen, and silicon. A number of these lines were not detected before in H-deficient stars. They are listed in Table 2 and will be discussed in detail below. Since the SDSS data range up to about $10000 \AA$, they allow us to study the spectra at wavelengths beyond the hitherto observed $\mathrm{H}_{\alpha}$ region. We detected several lines of the CNO elements (Fig. 3, Table 2).

\section{Spectral analysis}

A grid of line-blanketed non-local thermodynamic equilibrium (non-LTE) model atmospheres consisting of $\mathrm{H}, \mathrm{He}$, and $\mathrm{C}$ was computed, which are described in detail in Werner et al. (2004). It spans $T_{\text {eff }}=60-140 \mathrm{kK}$ and $\log g=4.8-8.3$, with steps of 5 or $10 \mathrm{kK}$ and 0.3 dex in the vicinity of the best fitting model of each star. $\mathrm{C} / \mathrm{He}$ number abundance ratios in the range 0 0.33 were considered. Oxygen was included in the models for the two stars exhibiting $\mathrm{O}$ lines. A larger $\mathrm{O}$ model atom was used for subsequent line formation calculations (keeping fixed the atmospheric structure). SDSS 1728 is the only star of our sample showing $\mathrm{Si}$ and $\mathrm{N}$ lines. Line formation calculations were carried out for these species in order to determine abundances. All model atoms were built from the publicly available Tübingen Model Atom Database ${ }^{2}$. Synthetic spectra were folded with Gaussians ( $F W H M=2.5 \AA$ ) accounting for the spectral resolution of the observations. The best fitting models were chosen by visual comparison with the rectified observed WD spectra.

2 http://astro.uni-tuebingen.de/ TMAD/ 
K. Werner et al.: New hydrogen-deficient (pre-) white dwarfs in the SDSS DR 10

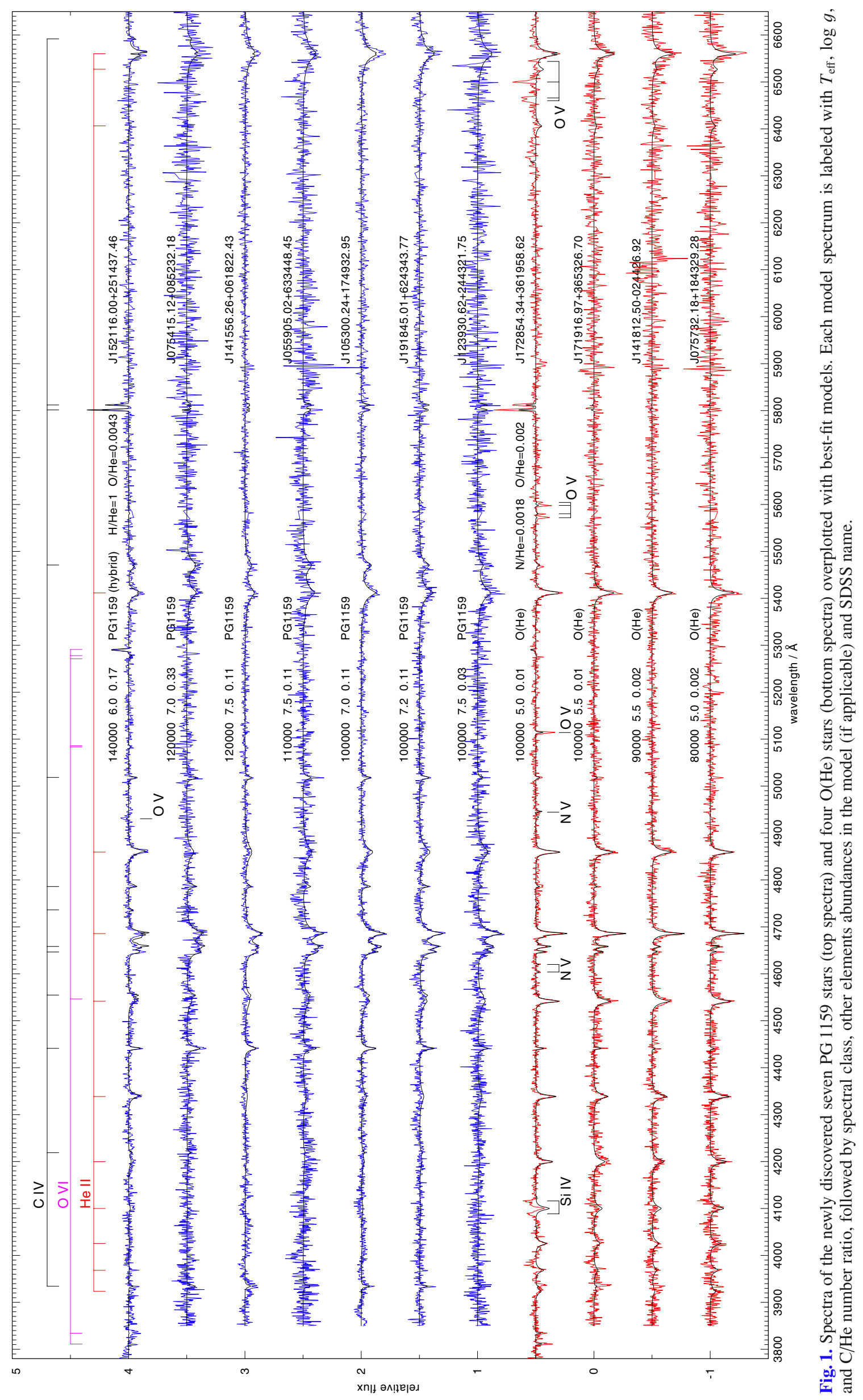

A53, page 3 of 8 
A\&A 564, A53 (2014)

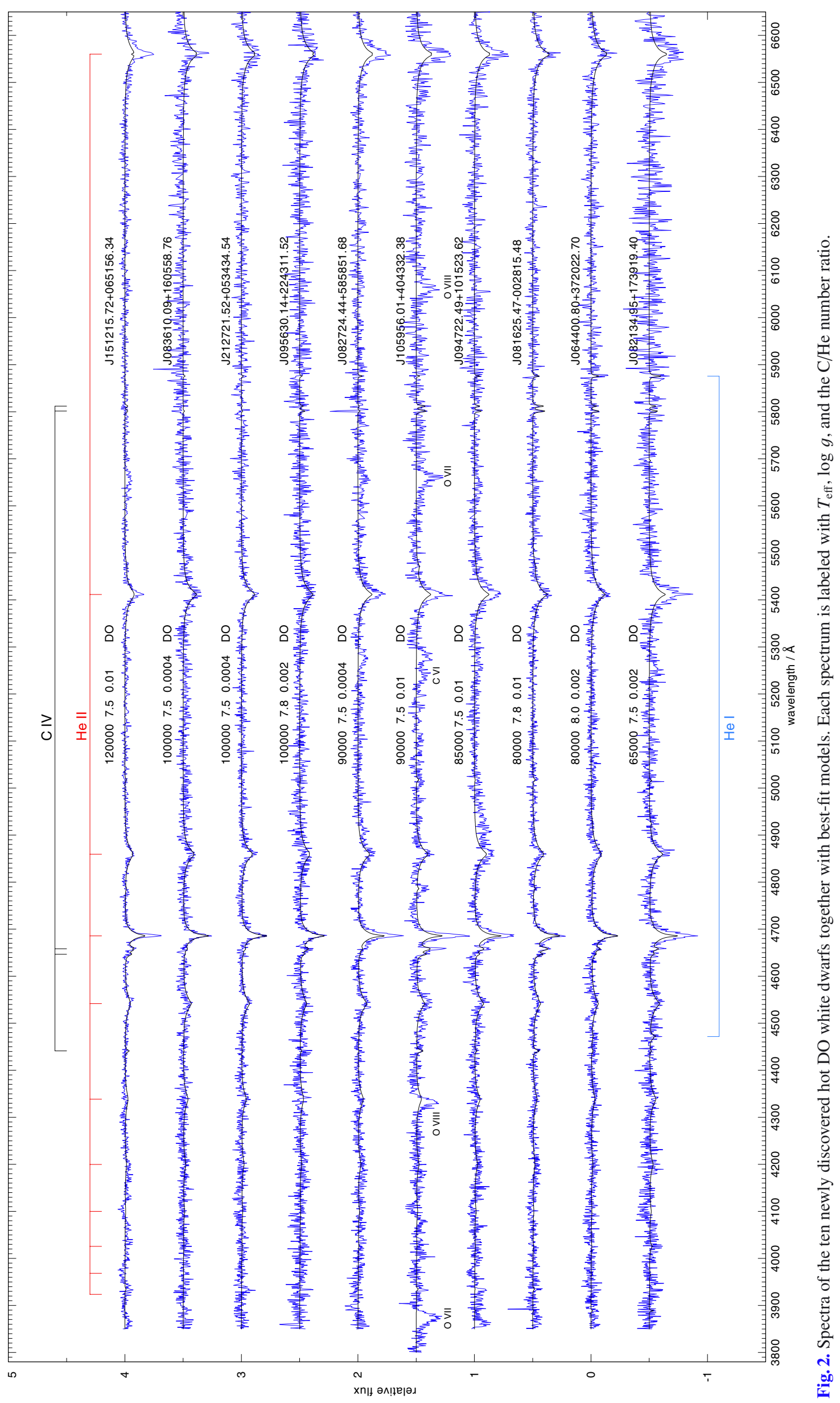


Table 2. Newly identified lines in H-deficient stars, as seen in the spectra of SDSS 1521 (PG 1159-type) and/or SDSS 1728 (O(He)-type).

\begin{tabular}{|c|c|c|}
\hline Wavelength/Å & Ion & Transition \\
\hline 4088.86 & Si IV & $4 \mathrm{~s}^{2} \mathrm{~S}_{1 / 2}-4 \mathrm{p}^{2}{ }^{2} \mathrm{P}_{3 / 2}^{\mathrm{o}}$ \\
\hline 4116.10 & Si IV & $4 \mathrm{~s}^{2} \mathrm{~S}_{1 / 2}-4 \mathrm{p}^{2}{ }^{2} \mathrm{P}_{1 / 2}^{\mathrm{o}}$ \\
\hline 4930.27 & $\mathrm{Ov}$ & $6 \mathrm{~h}{ }^{1} \mathrm{H}_{5}^{\mathrm{o}}-7 \mathrm{i}^{1} \mathrm{I}_{6}$ \\
\hline 5114.06 & $\mathrm{Ov}$ & $3 \mathrm{~s}^{1} \mathrm{~S}_{0}-3 \mathrm{p}^{2}{ }^{1} \mathrm{P}_{1}^{\mathrm{o}}$ \\
\hline 5571.81 & $\mathrm{Ov}$ & $3 \mathrm{p}^{3} \mathrm{P}_{0}^{\mathrm{o}}-3 \mathrm{~d}^{3} \mathrm{D}_{1}$ \\
\hline 5580.12 & $\mathrm{Ov}$ & $3 p^{3} P_{1}^{o}-3 d^{3} D_{2}$ \\
\hline 5597.89 & $\mathrm{OV}$ & $3 p^{3} P_{2}^{o}-3 d^{3} D_{3}$ \\
\hline 5604.27 & $\mathrm{Ov}$ & $3 p^{3} P_{2}^{o}-3 d^{3} D_{2}$ \\
\hline 5614.8: & $\mathrm{OV}$ & $4 \mathrm{~s}^{1} \mathrm{P}^{\mathrm{o}}-6 \mathrm{~d}^{1} \mathrm{D}$ \\
\hline 6460.1 & $\mathrm{OV}$ & $3 p^{3} D_{1}-3 d^{2}{ }^{3} F_{2}^{o}$ \\
\hline 6466.1 & $\mathrm{OV}$ & $3 p^{3} D_{2}-3 d^{2}{ }^{3} F_{3}^{2}$ \\
\hline 6500.2 & $\mathrm{OV}$ & $3 p^{3} D_{3}-3 d^{2}{ }^{3} F_{4}^{o}$ \\
\hline 6543.8: & $\mathrm{OV}$ & $3 p^{3} D_{2}-3 d^{2}{ }^{3} F_{2}^{0}$ \\
\hline 6683.20 & He II & $5-13$ \\
\hline 6890.90 & He II & 5-12 \\
\hline 7050.8 & CiV & $7 \mathrm{~d}^{2} \mathrm{D}_{*}-9 \mathrm{f}^{2} \mathrm{~F}_{*}^{\mathrm{o}}$ \\
\hline 7061.2 & C IV & $7 f^{2} F_{*}^{o}-9 g^{2} G_{*}$ etc. \\
\hline 7177.52 & He II & $5-11$ \\
\hline 7379.6 & CiV & $6 \mathrm{p}^{2} \mathrm{P}_{1 / 2}^{\mathrm{o}}-7 \mathrm{~d}^{2} \mathrm{D}_{3 / 2}$ \\
\hline 7381.6 & CIV & $6 \mathrm{p}^{2} \mathrm{P}_{3 / 2}^{\mathrm{o}}-7 \mathrm{~d}^{2} \mathrm{D}_{*}$ \\
\hline 7422.4 & $\mathrm{Ov}$ & $4 s^{3} S_{1}-4 p^{2}{ }^{3} P_{2}^{o}$ \\
\hline 7437.3 & $\mathrm{Ov}$ & $4 s^{3} S_{1}-4 p^{2}{ }^{3} P_{1}^{o}$ \\
\hline 7443.1 & $\mathrm{Ov}$ & $4 s^{3} S_{1}-4 p^{2}{ }^{3} P_{0}^{0}$ \\
\hline 7592.75 & He II & $5-10$ \\
\hline 7618.7 & $\mathrm{~N} \mathrm{~V}$ & $7 \mathrm{i}^{2} \mathrm{I}_{*}-8 \mathrm{k}^{2} \mathrm{~K}_{*}^{\mathrm{o}}$ \\
\hline 7706.61 & CIV & $6 d^{2} \mathrm{D}_{3 / 2}-7 \mathrm{f}^{2} \mathrm{~F}_{5 / 2}^{\mathrm{o}}$ \\
\hline 7707.33 & CIV & $6 d^{2} \mathrm{D}_{5 / 2}-7 \mathrm{f}^{2} \mathrm{~F}_{*}^{\mathrm{o}}$ \\
\hline 7717 & $?$ & O VI $n=8-9 ?$ \\
\hline 7723.9 & C IV & $6 \mathrm{f}^{2} \mathrm{~F}_{*}^{\mathrm{o}}-7 \mathrm{~g}{ }^{2} \mathrm{G}_{*}$ etc. \\
\hline 7948.6: & C IV & $6 \mathrm{~d}^{2} \mathrm{D}_{*}-7 \mathrm{p}^{2} \mathrm{P}_{*}^{\mathrm{o}}$ \\
\hline 8236.79 & He II & $5-9$ \\
\hline 8381.8: & C IV & $6 \mathrm{p}^{2} \mathrm{P}_{*}^{\mathrm{o}}-7 \mathrm{~s}^{2} \mathrm{~S}_{*}$ \\
\hline
\end{tabular}

Notes. Colons denote uncertain identifications. This table augments the line list of Werner et al. (1991). Wavelengths taken from the Kentucky Database v2.05b16: http://www.pa.uky.edu/ peter/newpage/

All observed spectra were shifted to rest-wavelengths by applying radial-velocity corrections as measured by centroiding the He II $\lambda 4686$ line and listed in Table 3. They are uncorrected for gravitational redshift.

The results of our analysis are summarized in Table 1 . The best fit model spectra are compared to the observations in Figs. 1 and 2, and in more detail in Figs. 3 and 4. The position of the stars in the $T_{\text {eff }}-\log g$ diagram is shown in Fig. 5. Error estimates for $T_{\text {eff }}$ and $\log g$ are given in Table 1. The errors for metal abundances are estimated to $\pm 0.5 \mathrm{dex}$. It is worthwhile to comment on some individual objects and to highlight difficulties encountered in our analysis.

\subsection{PG 1159 stars}

All PG 1159 stars except of SDSS 1521 are in the $T_{\text {eff }}$ range $100000-120000 \mathrm{~K}$ with $\log g=7.0-7.5$. The derived $\mathrm{C}$ abundances are relatively low (see Werner \& Herwig for a compilation of atmospheric parameters and references therein). With the exception of SDSS $0754(\mathrm{C} / \mathrm{He}=0.33)$, the abundance range is $\mathrm{C} / \mathrm{He}=0.03-0.17$, while previous analyses revealed many stars with $\mathrm{C} / \mathrm{He}=0.5$. SDSS 1239 has the lowest $\mathrm{C}$ abundance (0.03). It is identical to the lowest value found previously for a PG 1159

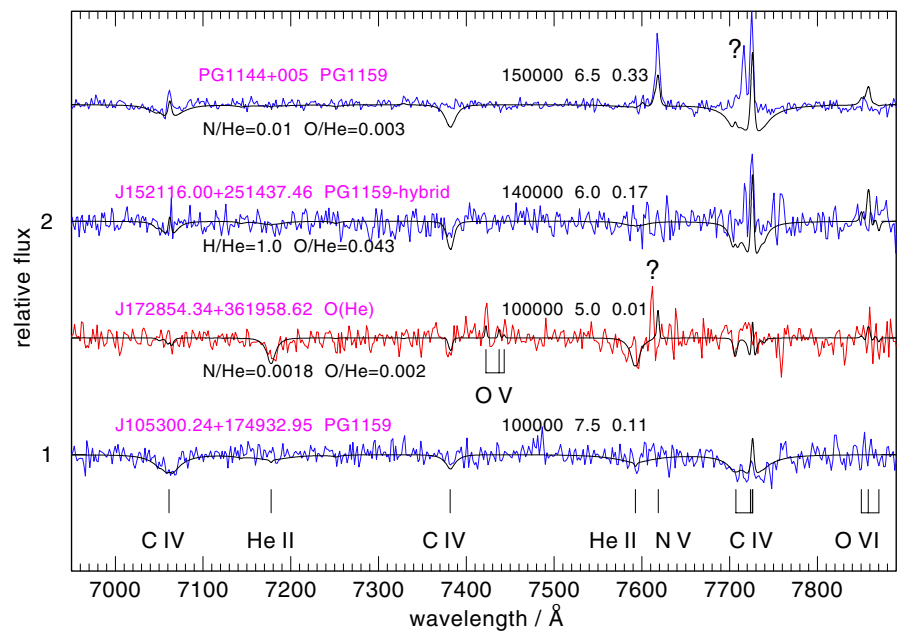

Fig. 3. Red parts of the SDSS spectra of three selected program stars (lower spectra). The upper SDSS spectrum is from a known PG 1159 star (PG 1144+005). The O VI emission feature at $7870 \AA$ in the models is possibly related to the unidentified emission feature at $7717 \AA$. Each spectrum is labeled with $T_{\text {eff }}, \log g, \mathrm{C} / \mathrm{He}$ number ratio of the overplotted model. If applicable, other element abundances in the models are indicated, too.

Table 3. Radial velocity corrections applied to the observed spectra.

\begin{tabular}{lr}
\hline \hline Star [SDSS J] & $v_{\mathrm{rad}}\left[\mathrm{km} \mathrm{s}^{-1}\right]$ \\
\hline $055905.02+633448.45$ & 0 \\
$064400.80+372022.70$ & 100 \\
$075415.12+085232.18$ & 140 \\
$075732.18+184329.28$ & 60 \\
$081625.47-002815.48$ & 70 \\
$082134.95+173919.40$ & 90 \\
$082724.44+585851.68$ & 80 \\
$083610.09+160558.76$ & 30 \\
$094722.49+101523.62$ & 130 \\
$095630.14+224311.52$ & 80 \\
$105300.24+174932.95$ & 80 \\
$105956.01+404332.38$ & 60 \\
$123930.62+244321.75$ & 50 \\
$141556.26+061822.43$ & 140 \\
$141812.50-024426.92$ & 0 \\
$151215.72+065156.34$ & 90 \\
$152116.00+251437.46$ & 40 \\
$171916.97+365326.70$ & -160 \\
$172854.34+361958.62$ & -280 \\
$191845.01+624343.77$ & 100 \\
$212721.52+053434.54$ & 60 \\
\hline
\end{tabular}

star (Hügelmeyer et al. 2006) and it is rather close to the maximum $\mathrm{C}$ abundance of 0.01 found in previously analyzed DOs and four DOs of our sample (see below). The spectral-class distinction between such DOs and PG 1159 stars with respect to their $\mathrm{C}$ abundances is therefore somewhat arbitrary because the $\mathrm{C}$-abundance gap is rather small. We suggest to set this limit at $\mathrm{C} / \mathrm{He}=0.02$ in order to conserve previously assigned classes.

SDSS 1521 is a particularly interesting object and deserves a detailed discussion of its spectrum and the line-profile modeling. It is a hybrid-PG 1159 star, i.e. it has residual hydrogen in its atmosphere. This is immediately visible when comparing the relatively weak He II $\lambda 5410$ with the stronger adjacent He II $\lambda 4340$ and He II $\lambda 4860$ lines that are blended by $\mathrm{H}_{\gamma}$ and 

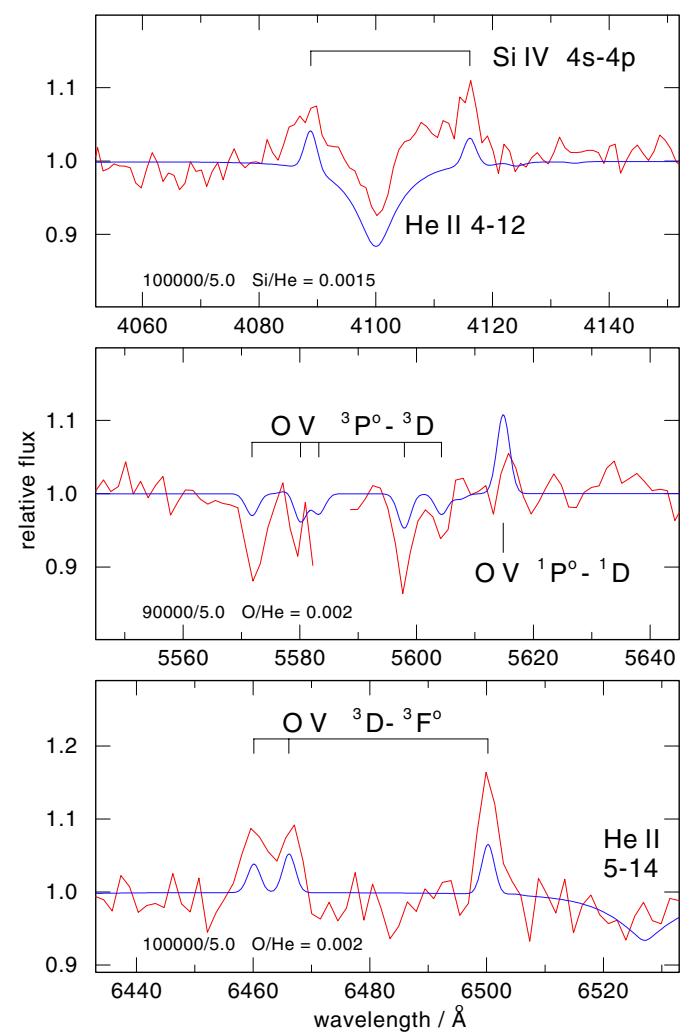

Fig. 4. Details of the spectrum from the new $\mathrm{O}(\mathrm{He})$ star SDSS 1728 centered on particular Si IV and OV multiplets. Overplotted are model spectra with $T_{\text {eff }}, \log g$, as well as $\mathrm{Si}$ and $\mathrm{O}$ abundances as given in the panels. The $\mathrm{C} / \mathrm{He}$ ratio in the models is $1 \%$.

$\mathrm{H}_{\beta}$, respectively. The hybrids are thought to be created by a particular variant of late He-shell flashes, the AGB final thermal puls (AFTP) scenario. Only four such objects were hitherto known, all of them being cooler (96000-130000 K; see Werner $\&$ Herwig 2006, and references therein). The surface gravity of SDSS 1521 is rather low $(\log g=6)$ but not unusual, as the other four hybrids known have $\log g=6.8-5.7$.

The $\mathrm{H} / \mathrm{He}$ ratio in our hybrid star is unity, compared to $\mathrm{H} / \mathrm{He}=2$ determined for three hybrid-PG 1159s (Dreizler 1998). It also exhibits a strong emission line of O VI $\lambda 5293$ due to its high effective temperature $(140000 \mathrm{~K})$ and a weaker one from $\mathrm{O} v \lambda 4930$ that allow us to constrain the $\mathrm{O}$ abundance. We find $\mathrm{O} / \mathrm{He}=0.043$, a value that is similar to that established for the mentioned three other hybrids $(\mathrm{O} / \mathrm{He}=0.04)$. These oxygen lines as well as the $\mathrm{C}$ IV $\lambda 5802 / 12$ doublet are sensitive temperature indicators. While the $\mathrm{O}$ lines disappear when $T_{\text {eff }}$ decreases below about $120000 \mathrm{~K}$, the C IV doublet turns from emission into absorption (this behavior depends in detail on gravity). We also see the O VI $\lambda 3811 / 34$ doublet. The strongest line $(6 h-7 i)$ of the C IV $n=6-7$ transition at $7726.3 \AA$ is in emission (Fig. 3). The adjacent emission feature at $7717 \AA$ remains unidentified. A possible candidate are the high-1 O VI $n=8-9$ transition components. In our model, a respective emission feature appears at $7858 \AA$ (according to NIST $^{3}$ energy levels) but at this position there is no line in the observed spectrum. According to the Kentucky database, that O VI line is at $7740 \pm 53 \AA$, which would be consistent with the observed feature at $7717 \AA$.

All CIV lines in the model match the observation of SDSS 1521 well, except for the trough at $7724 \AA$ that is formed

\footnotetext{
3 http://physics.nist.gov/
}

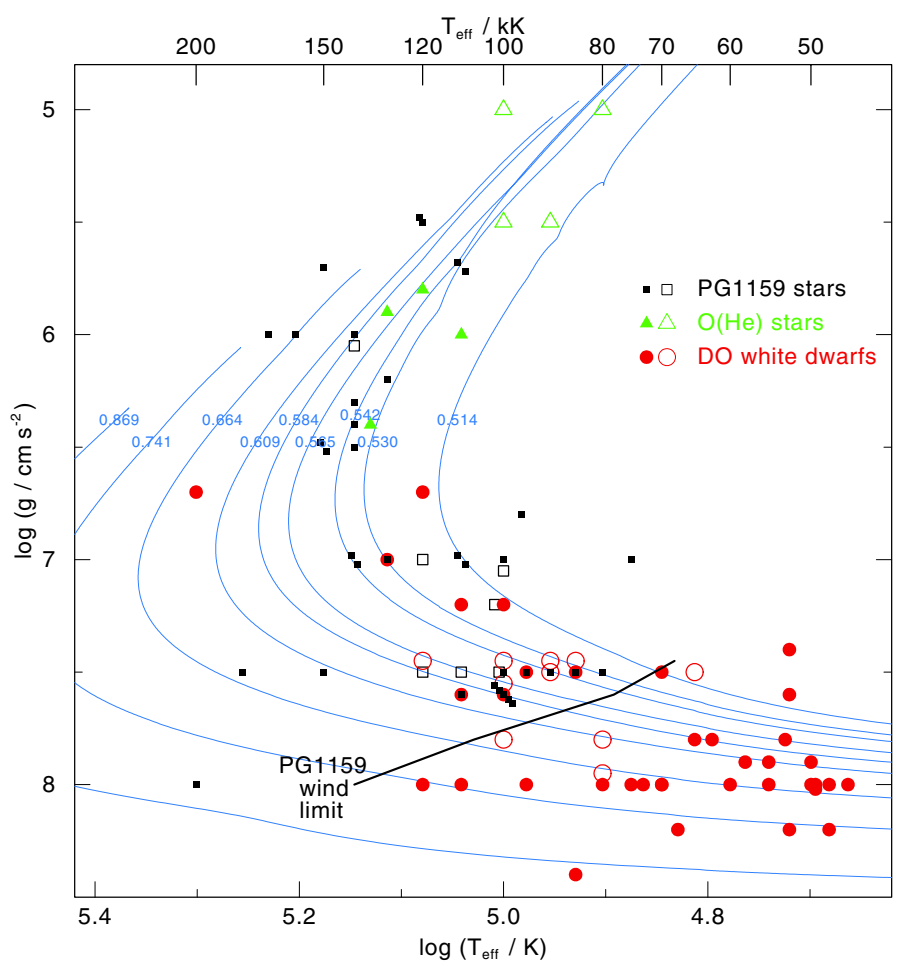

Fig. 5. Position of the PG 1159 stars, $\mathrm{O}(\mathrm{He})$ stars, and hot DOs analyzed in this paper in the $g-T_{\text {eff }}$ diagram (open symbols). Full symbols denote previously analyzed hot and cool DOs as well as PG 1159 and $\mathrm{O}(\mathrm{He})$ stars. Evolutionary tracks for H-deficient WDs (Althaus et al. 2009) are labeled with the respective stellar mass in $M_{\odot}$. Also shown is one of the theoretical wind limits from Unglaub \& Bues (2000) near which PG 1159 stars transform into DOs because gravitational settling overcomes radiation-driven mass loss.

by the high-1 components of the $n=6-7$ transition. While the central emission peak matches well, the line wings in the model are too deep (Fig. 3). We further investigated this problem by looking at the spectrum of the hot PG 1159 star PG 1144+005 $\left(T_{\text {eff }}=150000 \mathrm{~K}, \log g=6.5\right.$; Werner \& Heber 1991), taken from the SDSS DR10 and shown in the top of Fig. 3. Here the discrepancy between model and observation is even more obvious and it also affects other C IV 6-7 components, namely 6p$7 \mathrm{~d}$ at $7382 \AA$ and $6 \mathrm{~d}-7 \mathrm{p}$ at $7949 \AA$. We have no explanation for this mismatch, in particular because the 5-6 lines (responsible for the $4648 / 60 \AA$ trough and most other lines in the optical), the 7-9 lines (responsible for the 7061 8 trough), and the 68 lines (e.g. at 4736 and $4788 \AA$ ) are all well matched, and in past work we never encountered fitting problems for a variety of C IV lines in the UV region. The inability of the models to fit the C IV 6-7 transitions seems to be restricted to relatively lowgravity stars. At the bottom of Fig. 3, we display the spectrum of another PG 1159 star from our sample (SDSS 1053) that has $\log g=7.5$ and which is fit reasonably well by the model at the $7724 \AA$ trough.

\section{2. $\mathrm{O}(\mathrm{He})$ stars}

The four discovered $\mathrm{O}(\mathrm{He})$ stars are easily discernible from the DO white dwarfs and PG 1159 stars by their relatively narrow He II lines such that the Pickering series members can be seen down to $\approx 4000 \AA$. They have similar temperatures and gravities $\left(T_{\text {eff }}=80000 \mathrm{~K}-100000 \mathrm{~K}, \log g=5.0-5.5\right)$ and show traces of carbon $(\mathrm{C} / \mathrm{He}=0.002-0.01)$. 
SDSS 1728 is remarkable because it displays many $\mathrm{O} v$ lines besides O VI $\lambda 3811 / 34$. We see a singlet at $\lambda 5114$, at $\lambda \lambda 5572-$ 5604 the strongest members of a multiplet in absorption, and at $\lambda \lambda 6460-6544$ the strongest lines of another multiplet in emission (see also Fig. 4). In addition, the $\mathrm{O} v \lambda \lambda 7422 / 37 / 43$ triplet is found in emission (Fig. 3). The star also exhibits nitrogen lines, namely the $\mathrm{N} v \lambda \lambda 4604 / 20$ doublet and a very weak $\mathrm{N} v \lambda 4945$ line (Fig. 1). The emission feature at $7612 \AA$ (Fig. 3) is about $7 \AA$ blueward of the $\mathrm{N} \mathrm{V} \lambda 7618.77 \mathrm{i}-8 \mathrm{k}$ line that is visible in PG 1144+005 and which, based on our model, is expected to be present in SDSS 1728. This blueshift remains unexplained. For the first time in any hot H-deficient star, we discover optical silicon lines; the Si IV $\lambda \lambda 4089 / 4116$ doublet that appears in emission (Fig. 4). The metal abundances derived from these features are summarized in Table 1.

It is interesting to compare the properties of our four new $\mathrm{O}(\mathrm{He})$ stars to those of the previously known four ones, that were recently re-analyzed by Reindl et al. (2014). The latter have $T_{\text {eff }}=110000-135000 \mathrm{~K}, \log g=5.8-6.4$, i.e. they are all hotter and have higher gravities, hence they are more evolved (see also Fig.5). Concerning their $\mathrm{C}$ and $\mathrm{N}$ abundances it is notable that three of the Reindl et al. sample stars are N-rich $\left(\mathrm{N} / \mathrm{He}=4-40 \times 10^{-4}\right)$ and $\mathrm{C}$-poor $\left(\mathrm{C} / \mathrm{He}<2 \times 10^{-4}\right)$ while one star shows carbon $(\mathrm{C} / \mathrm{He}=0.003)$ but no nitrogen $(\mathrm{N} / \mathrm{He}<$ $\left.3 \times 10^{-5}\right)$. The latter also holds for three of our $\mathrm{O}(\mathrm{He}) \mathrm{s}$. In contrast, SDSS 1728 from our sample is different because it has $\mathrm{C}$ and $\mathrm{N}$ present $(\mathrm{C} / \mathrm{He}=0.01, \mathrm{~N} / \mathrm{He}=0.0018)$. In addition, it has measurable oxygen, $\mathrm{O} / \mathrm{He}=0.002$, which is significantly higher than even the largest upper limit of the Reindl et al. all sample stars $\left(3 \times 10^{-4}\right)$. The same holds for silicon $(\mathrm{Si} / \mathrm{He}=0.0015$ compared to $\left.\mathrm{Si} / \mathrm{He}<1.4 \times 10^{-4}\right)$. In conclusion, SDSS 1728 is the $\mathrm{O}(\mathrm{He})$ star with the richest $\mathrm{CNO}$ and $\mathrm{Si}$ composition.

The oxygen lines in SDSS 1728 are very temperature sensitive. Although each single one can be fitted with a particular model from a very narrow $T_{\text {eff }}$ range $( \pm 5000 \mathrm{~K})$, it is not possible to find a single model that fits all $\mathrm{O}$ lines simultaneously. For example, the very weak O VI $\lambda 5293$ emission is only fitted by the $100000 \mathrm{~K}$ model that is shown in Fig. 1. Increasing or decreasing the temperature results in a too strong emission line in the model or it completely vanishes, respectively. At the same temperature, the observed O V absorption line at $5114 \AA$ and the emission multiplet at $\lambda \lambda 7422-7443 \AA$ are fit quite well. On the other hand, that model has a too weak O v $\lambda \lambda$ 5571-5604 absorption multiplet, which is better matched by a $90000 \mathrm{~K}$ model, see Fig. 4. In the same panel of that figure it is seen, however, that another O V line at $5615 \AA$ is in strong emission at $90000 \mathrm{~K}$, in contrast to the observation that shows this line in weak emission at best. The observed O v $\lambda \lambda 6460-6500$ emission multiplet could only be reproduced satisfactorily with a $100000 \mathrm{~K}$ model (Fig. 4) after we considerably increased the size of the $\mathrm{O} V$ model atom from 34 to 90 energy levels.

\subsection{DO white dwarfs}

Our model spectra in Fig. 2 show that the He I lines disappear in DOs with $T_{\text {eff }} \gtrsim 90000 \mathrm{~K}$. It is clear that the distinction between hot and cool DOs depends on the quality of the spectra and we therefore have DOs down to $T_{\text {eff }}=65000 \mathrm{~K}$ with relatively poor $\mathrm{S} / \mathrm{N}$ in our selected sample. The hottest $\mathrm{DO}$ we found has $T_{\text {eff }}=120000 \mathrm{~K}$. Surface gravities range between $\log g=$ 7.5-8. Seven out of the ten DOs show C IV lines and we derive $\mathrm{C} / \mathrm{He}=0.002-0.01$. Upper limits of $\mathrm{C} / \mathrm{He}=0.0004$ were found for the remaining three. Carbon abundances in DOs up to the $1 \%$ level are quite rare but have been detected in two objects before (Dreizler \& Werner 1996).

While we achieve good model fits for five of our ten DOs, we encountered problems with the others which have such deep He II lines that they cannot be fitted by any model. In particular, SDSS 1059 is a new case of the still enigmatic group of hot WDs that exhibit extraordinary absorption lines ascribed to ultra-high ionized elements, e.g. C VI, O VII, and O VIII. Such ionization stages cannot be thermally excited in the photosphere. Since the strongest such lines often show a rather broad blue wing it was speculated that they are formed in a stellar wind with perhaps shock-heated regions. This phenomenon is not restricted to hot DO WDs, where it was detected first (Werner et al. 1995) but it is was also seen in a DAO WD (Dreizler et al. 1995) and in a PG 1159 star (Hügelmeyer et al. 2006). The formation process of these extraordinary lines obviously affects the photospheric line profiles of He II (and C IV $\lambda$ 4648/60) in such a way that they become so deep that they cannot be matched by any usual model.

The other four problematic DOs (SDSS 1512, SDSS 0827, SDSS 0947, SDSS 0821) have similarly strong He II lines like that "hot-wind" DO SDSS 1059, but they do not exhibit the ultrahigh ionization features, except for a possible broad and weak depression at $5670 \AA$. One may speculate that the same unknown process is at work here, affecting the He II lines but not generating the strong ultra-high ionization lines.

How do we constrain the atmospheric parameters in these problematic cases, where no information can be obtained from the entire set of He II lines? A lower limit to $T_{\text {eff }}$ can be inferred from the absence of He I lines. In those cases where we see the C IV $\lambda$ 5802/12 doublet we can further constrain $T_{\text {eff }}$, however, only to limited extent because its strength also depends on the $\mathrm{C}$ abundance. The latter can be constrained by C IV $\lambda 4440$ that is less temperature dependent. C IV $\lambda 4648 / 60$ is a problem, too. It also seems to be "too deep" like e.g. He II $\lambda 4686$. A lower limit to the surface gravity is estimated from the absence of Pickering lines below $4500 \AA$. Limits for $\log g$ are also drawn from He II $\lambda 4860$ and $\lambda 4540$, which are relatively weak, form deep in the atmosphere and are thus perhaps least affected by the process that creates the too-deep He II lines.

\section{Summary and conclusions}

In the SDSS DR10 we have discovered 21 new hot hydrogendeficient (pre-) white dwarfs and performed a spectral analysis with non-LTE model atmospheres. We found seven PG 1159 stars. Ten new hot DO white dwarfs were detected, from which six have $T_{\text {eff }} \geq 90000 \mathrm{~K}$ as compared to just nine previously known DOs with such high temperature. Our analysis confirms the evolutionary link between PG 1159 stars and the subsequent DO white dwarfs (Fig. 5). The PG 1159 stars have $\mathrm{C} / \mathrm{He}=$ 0.33 to 0.03 , whereas the DOs have lower carbon abundances $(\mathrm{C} / \mathrm{He} \leq 0.01)$ as a consequence of advanced gravitational settling. One of the PG 1159 stars represents the fifth member of the hybrid-PG 1159 subclass, i.e., it shows residual hydrogen. Consequently, this star must evolve to a DA white dwarf.

We identified four new $\mathrm{O}(\mathrm{He})$ stars, doubling the number of members of this spectral class. These stars are pre-WDs, heliumdominated with only trace amounts of carbon. Their origin must be different from the PG 1159 stars, because the late He-shell flash scenario always produces objects with much higher $\mathrm{C}$ abundances. Possible explanations are discussed at length by Reindl et al. (2014) in their analysis of the previously known 
$\mathrm{O}(\mathrm{He})$ stars and must not be repeated in detail here. It suffices to state that their origin is mysterious and that they are representing a second $\mathrm{H}$-deficient evolutionary channel leading to DO white dwarfs.

One of our O(He) stars (SDSS 1728) stands out in the group of now in total eight objects because it has the highest $\mathrm{CNO}$ and $\mathrm{Si}$ abundances. In terms of mass fractions, $\mathrm{O}=7.7 \times 10^{-3}$ is about solar (within analysis errors) while $\mathrm{C}=2.9 \times 10^{-2}$ and $\mathrm{N}=6 \times 10^{-3}$ are clearly overabundant (twelve and nine times solar, respectively). Such a CNO abundance pattern results from a particular variant of a binary He-white dwarf merger (so-called composite merger model) as studied by Zhang \& Jeffery (2012). The silicon abundance in SDSS $1728(\mathrm{Si}=0.01)$ is strongly overabundant, too (15 times solar), but no predictions of merger models are available for comparison.

The mean mass of our ten DOs (Table 1) is $0.57_{-0.06}^{+0.16} M_{\odot}$, which is in good agreement with the mean value of $0.59 M_{\odot}$ derived for 14 DOs by Dreizler \& Werner (1996). Hügelmeyer et al. (2006) already noted that the mean value of their sample of 20 DOs from the SDSS DR4 was somewhat larger $\left(0.69 M_{\odot}\right)$, although some of the difference could be due to the calibration problem reported in Kleinman et al. (2009, 2013). The mean mass of our new PG 1159 stars is $0.54_{-0.03}^{+0.09} M_{\odot}$, which is in good agreement with the value derived from all (36) previously analyzed PG 1159 stars $\left(0.57 M_{\odot}\right.$, Miller Bertolami \& Althaus 2006). The mean mass of our four $\mathrm{O}(\mathrm{He})$ stars is almost identical to that of our DOs $\left(0.58_{-0.05}^{+0.18} M_{\odot}\right)$. Three of them are of relatively low mass $\left(0.52-0.53 M_{\odot}\right)$ which agrees very well with the mass range of the previously analyzed $\mathrm{O}(\mathrm{He})$ stars when the same post-AGB tracks are used as in our analysis $(0.52-$ $0.57 M_{\odot}$; Reindl et al. 2014). In conclusion, there is no significant difference in the mean masses of the three considered H-deficient spectral classes.

Finally, we stress that we could not obtain satisfying line profile fits for $50 \%$ of the DOs because they have unusually deep He II lines. The unknown origin of this mismatch is probably related to the occurrence of another phenomenon, namely the appearance of ultra-high ionization lines (e.g. for O VII and O VIII) in one of these DOs. This phenomenon is now known since almost two decades when in was discovered for the first time (Werner et al. 1995). It is affecting a large fraction of the known hot DOs and awaits an explanation.
Acknowledgements. T. Rauch is supported by the German Aerospace Center (DLR) under grant 05 OR 1301. Kepler is supported by CNPq-Brazil. This research has made use of the SIMBAD database, operated at CDS, Strasbourg, France. Funding for SDSS-III has been provided by the Alfred P. Sloan Foundation, the Participating Institutions, the National Science Foundation, and the US Department of Energy Office of Science. The SDSS-III web site is http://www.sdss3.org/. SDSS-III is managed by the Astrophysical Research Consortium for the Participating Institutions of the SDSS-III Collaboration including the University of Arizona, the Brazilian Participation Group, Brookhaven National Laboratory, Carnegie Mellon University, University of Florida, the French Participation Group, the German Participation Group, Harvard University, the Instituto de Astrofisica de Canarias, the Michigan State/Notre Dame/JINA Participation Group, Johns Hopkins University, Lawrence Berkeley National Laboratory, Max Planck Institute for Astrophysics, Max Planck Institute for Extraterrestrial Physics, New Mexico State University, New York University, Ohio State University, Pennsylvania State University, University of Portsmouth, Princeton University, the Spanish Participation Group, University of Tokyo, University of Utah, Vanderbilt University, University of Virginia, University of Washington, and Yale University.

\section{References}

Althaus, L. G., Panei, J. A., Miller Bertolami, M. M., et al. 2009, ApJ, 704, 1605

Dreizler, S. 1998, Baltic Astron., 7, 71

Dreizler, S., \& Werner, K. 1996, A\&A, 314, 217

Dreizler, S., Heber, U., Napiwotzki, R., \& Hagen, H. J. 1995, A\&A, 303, L53

Hügelmeyer, S. D., Dreizler, S., Werner, K., et al. 2005, A\&A, 442, 309

Hügelmeyer, S. D., Dreizler, S., Homeier, D., et al. 2006, A\&A, 454, 617

Kleinman, S. J., Nitta, A., \& Koester, D. 2009, J. Phys. Conf. Ser., 172, 2020

Kleinman, S. J., Kepler, S. O., Koester, D., et al. 2013, ApJS, 204, 5

Miller Bertolami, M. M., \& Althaus, L. G. 2006, A\&A, 454, 845

Rauch, T., Werner, K., Biémont, É., Quinet, P., \& Kruk, J. W. 2012, A\&A, 546, A55

Rauch, T., Werner, K., Bohlin, R., \& Kruk, J. W. 2013, A\&A, 560, A106

Reindl, N., Rauch, T., Werner, K., Kruk, J. W., \& Todt, H. 2014, A\&A, submitted

Unglaub, K., \& Bues, I. 2000, A\&A, 359, 1042

Wassermann, D., Werner, K., Rauch, T., \& Kruk, J. W. 2010, A\&A, 524, A9

Werner, K., \& Heber, U. 1991, A\&A, 247, 476

Werner, K., \& Herwig, F. 2006, PASP, 118, 183

Werner, K., Heber, U., \& Hunger, K. 1991, A\&A, 244, 437

Werner, K., Dreizler, S., Heber, U., et al. 1995, A\&A, 293, L75

Werner, K., Rauch, T., Reiff, E., Kruk, J. W., \& Napiwotzki, R. 2004, A\&A, 427, 685

Werner, K., Rauch, T., Ringat, E., \& Kruk, J. W. 2012, ApJ, 753, L7

Zhang, X., \& Jeffery, C. S. 2012, MNRAS, 419, 452 